\title{
Changing the epidemiology of carbapenem-resistant Pseudomonas aeruginosa in a Brazilian teaching hospital: the replacement of São Paulo metallo- $\beta$-lactamase-producing isolates
}

\author{
Felipe Lira de Sá Cavalcanti ${ }^{1,2}$, Anna Carolina Soares Almeida ${ }^{1,2}$, Marinalda Anselmo Vilela \\ Marcia Maria Camargo de Morais ${ }^{1}$, Marcos Antonio de Morais Junior²/+
}

'Laboratório de Resistência Microbiana, Instituto de Ciências Biológicas, Universidade de Pernambuco, Recife, PE, Brasil

${ }^{2}$ Departamento de Genética, Universidade Federal de Pernambuco, Av. Prof. Moraes Rego 1235, 50670-901 Recife, PE, Brasil

In Brazil, carbapenem-resistant Pseudomonas aeruginosa isolates are closely related to the São Paulo metallo- $\beta$ lactamase (SPM) Brazilian clone. In this study, imipenem-resistant isolates were divided in two sets, 2002/2003 and 2008/2009, analysed by pulsed field gel electrophoresis and tested for the Ambler class B metallo- $\beta$-lactamase (MBL) genes $\mathrm{bla}_{S P M-P}, \mathrm{bla}_{I M P}$ and $\mathrm{bla}_{V I M}$. The results show a prevalence of one clone related to the SPM Brazilian clone in 2002/2003. In 2008/2009, P. aeruginosa isolates were mostly MBL negative, genetically diverse and unrelated to those that had been detected earlier. These findings suggest that the resistance to carbapenems by these recent $\mathrm{P}$. aeruginosa isolates was not due to the spread of MBL-positive SPM-related clones, as often observed in Brazilian hospitals.

Key words: carbapenemases - metallo- $\beta$-lactamase - Pseudomonas aeruginosa

Carbapenems, including imipenem and meropenem, have been considered for the treatment of Pseudomonas aeruginosa infections because they are not hydrolysed by most of the serine $\beta$-lactamases (Hurst \& Lamb 2000). However, the prevalence of carbapenem resistance in this bacterium has increased worldwide, particularly in Latin America (Sader et al. 2004). This resistance can be conferred by the absence of the outer membrane protein OprD with the associated over-expression of the efflux pump MexAB-OprM (as in the case of meropenem resistance), by increased action of chromosome-encoded AmpC cephalosporinase or by the production of Ambler class B metallo- $\beta$-lactamases (MBLs) (Zavascki et al. 2005, Rodríguez-Martínez et al. 2009, Strateva \& Yordanov 2009). Among the MBL genes, bla ${ }_{\mathrm{SPM}-1}$ has thus far only been detected in Brazil and Switzerland and the dissemination of this gene in various regions of the country seems to be caused by a single epidemic $P$. aeruginosa clone (Gales et al. 2003, Salabi et al. 2010). In the last few years, an increasing number of carbapenemresistant $P$. aeruginosa isolates have been observed in a teaching hospital in Recife, Pernambuco, Brazil. In light of this, we decided to conduct a phenotypic and genetic analysis of those isolates to investigate the production of MBL and establish their relationships to the São Paulo metallo- $\beta$-lactamase (SPM) Brazilian clone.

Imipenem/ceftazidime-resistant isolates of $P$. aeruginosa were recovered from patients undergoing treatment in the Oswaldo Cruz University Hospital during the time frames 2002-2003 and 2008-2009; one isolate

Financial support: CNPq, CAPES, FACEPE

+ Corresponding author: marcos.morais@pq.cnpq.br Received 7 July 2011

Accepted 29 November 2011 was used per patient for a total of 73 isolates. The bacterial species were identified by standard biochemical tests. Susceptibility testing was performed by means of the disk-diffusion method with the following antimicrobial agents: imipenem, ceftazidime, ciprofloxacin, amikacin, gentamicin, piperacillin/tazobactam, aztreonam and polymyxin $\mathrm{B}$, in compliance with the Clinical and Laboratory Standards Institute guidelines (CLSI 2010). The minimal inhibitory concentration (MIC) for carbapenems of MBL-negative isolates was determined by the automated BD Phoenix system and interpreted in accordance with CLSI. Selected isolates were screened for MBL production by the disk approximation test as previously described (Arakawa et al. 2000).

Presumptive MBL producers were further tested for the $b l a_{\mathrm{SPM}-1}, b l a_{\mathrm{IMP}}$ and $b l a_{\mathrm{VIM}}$ genes. Bacterial DNA was extracted by using the Brazol kit (LGC Biotecnologia, Brazil) following the recommendations of the manufacturer and analysed by polymerase chain reaction (PCR) using the primer pairs bla $a_{\mathrm{SPM}-1}$ (forward: 5'-CCTACAATCTAACGGCGACC-3', reverse: 5'TCGCCGTGTCCAGGTATAAC-3'), bla $a_{\text {IMP }}$ (forward: 5'-GGAATAGAGTGGCTTAATTCTC-3', reverse: 5'GTGATGCGTCYCCAAYTTCACT-3') and bla ${ }_{\mathrm{VIM}}$ (forward: 5'-TGCGCATTCGACCGACAATC-3', reverse: 5'-GTCGAATGCGCAGCACCAGG-3') (Migliavacca et al. 2002, Gales et al. 2003, Toleman et al. 2005). Positive controls for the $P$. aeruginosa bla ${ }_{\mathrm{SPM}-1}$, bla $_{\mathrm{IMP}}$ and bla ${ }_{\mathrm{VIM}}$ genes were kindly provided by Special Clinical Microbiology Laboratory and ALERTA Laboratory (São Paulo, Brazil). The amplicons were purified with the aid of a PCR purification kit (Promega Co, USA) and submitted to DNA sequencing by the platform of the Aggeu Magalhães Research Centre, Oswaldo Cruz Foundation, Recife. The nucleotide sequences were evaluated with the BioEdit ${ }^{\mathrm{TM}}$ program and analysed by on-line BLASTn at GenBank dataset (National Centre for Biotechnology 
TABLE

Microbiological and molecular characteristics of carbapenem-resistant Pseudomonas aeruginosa isolates

\begin{tabular}{|c|c|c|c|c|c|c|}
\hline \multirow[b]{2}{*}{$\begin{array}{l}\text { Isolate } \\
\text { number }\end{array}$} & \multicolumn{3}{|c|}{ Resistance profile } & \multirow[b]{2}{*}{$\begin{array}{l}\text { MBL } \\
\text { test }^{a}\end{array}$} & \multirow[b]{2}{*}{$\begin{array}{l}\text { MBL } \\
\text { gene }\end{array}$} & \multirow[b]{2}{*}{$\begin{array}{c}\text { PFGE } \\
\text { pattern }^{b}\end{array}$} \\
\hline & Resistance & $\begin{array}{l}\text { Intermediate } \\
\text { resistance }\end{array}$ & Susceptibility & & & \\
\hline \multicolumn{7}{|c|}{$2002 / 2003$} \\
\hline Ps 53 & cip, ami, gen & - & azt, pol B & + & SPM-1 & A \\
\hline Ps 71 & cip, ami, gen & azt & pol B & + & SPM-1 & A \\
\hline Ps 76 & cip, ami, gen & - & azt, pol B & + & SPM-1 & A1 \\
\hline Ps 85 & cip, ami, gen & - & azt, pol B & + & SPM-1 & A \\
\hline Ps 91 & cip, ami, gen & - & azt, pol B & + & SPM-1 & A \\
\hline Ps 144 & cip, ami, gen & - & pip/taz, azt, pol B & + & SPM-1 & A2 \\
\hline Ps 158 & cip, ami, gen, pip/taz & azt & pol B & + & SPM-1 & A \\
\hline Ps 159 & cip, ami, gen, pip/taz & azt & pol B & + & SPM-1 & A2 \\
\hline Ps 169 & cip, ami, gen & - & pip/taz, azt, pol B & + & SPM-1 & A2 \\
\hline Ps 172 & cip, ami, gen & azt & pip/taz, pol B & + & SPM-1 & A \\
\hline Ps 178 & cip, ami, gen & azt & pip/taz, pol B & + & SPM-1 & A \\
\hline Ps 199 & cip, ami, gen & azt & pip/taz, pol B & + & SPM-1 & A \\
\hline Ps 212 & cip, ami, gen & azt & pip/taz, pol B & + & SPM-1 & A \\
\hline Ps 215 & cip, ami, gen & azt & pip/taz, pol B & + & SPM-1 & A \\
\hline Ps 241 & cip, ami, gen & - & pip/taz, azt, pol B & + & SPM-1 & A \\
\hline Ps 246 & cip, ami, gen & - & pip/taz, azt, pol B & + & SPM-1 & A \\
\hline Ps 305 & cip, ami, gen & azt & pip/taz, pol B & + & SPM-1 & A \\
\hline Ps 307 & cip, ami, gen & azt & pip/taz, pol B & + & SPM-1 & A \\
\hline Ps 327 & cip, ami, gen & azt & pip/taz, pol B & + & SPM-1 & A \\
\hline Ps 370 & cip, ami, gen, pip/taz & azt & pol B & + & AT & A \\
\hline Ps 408 & cip, ami, gen, pip/taz & azt & pol B & + & AT & A \\
\hline Ps 444 & cip, ami, gen, pip/taz & azt & pol B & + & SPM-1 & A \\
\hline Ps 445 & cip, ami, gen, azt & - & pip/taz, pol B & + & SPM-1 & A \\
\hline Ps 467 & cip, ami, gen, pip/taz & - & azt, pol B & + & SPM-1 & A \\
\hline Ps 527 & cip, ami, gen, pip/taz & - & azt, pol B & + & SPM-1 & A \\
\hline Ps 581 & cip, ami, gen, pip/taz & azt & pol B & + & SPM-1 & A \\
\hline Ps 598 & cip, ami, gen, pip/taz & - & azt, pol B & + & SPM-1 & A \\
\hline Ps 603 & ami, gen & azt & pip/taz, pol B & + & SPM-1 & A \\
\hline Ps 614 & ami, gen & - & pip/taz, azt, pol B & + & SPM-1 & A \\
\hline Ps 616 & ami, gen & - & pip/taz, azt, pol B & + & SPM-1 & A \\
\hline Ps 645 & ami, gen & - & pip/taz, azt, pol B & + & SPM-1 & A \\
\hline Ps 646 & ami, gen & - & pip/taz, azt, pol B & + & SPM-1 & A \\
\hline Ps 647 & ami, gen & azt & pip/taz, pol B & + & SPM-1 & A \\
\hline Ps 655 & cip, ami, gen, pip/taz & azt & pol B & + & SPM-1 & A \\
\hline Ps 668 & cip, ami, gen, pip/taz & azt & pol B & + & SPM-1 & A \\
\hline Ps 678 & cip, ami, gen & azt & pip/taz, pol B & + & SPM-1 & A \\
\hline Ps 712 & cip, ami, gen & - & pip/taz, azt, pol B & + & SPM-1 & A \\
\hline Ps 724 & cip, ami, gen & azt & pip/taz, pol B & + & SPM-1 & A \\
\hline Ps 725 & cip, ami, gen, pip/taz & - & azt, pol B & + & SPM-1 & A \\
\hline Ps 727 & cip, ami, gen & azt & pip/taz, pol B & + & SPM-1 & A \\
\hline Ps 762 & ami, gen, pip/taz & azt & pol B & + & SPM-1 & A \\
\hline Ps 767 & cip, ami, gen & azt & pip/taz, pol B & + & SPM-1 & A \\
\hline Ps 773 & ami, gen & azt & pip/taz, pol B & + & SPM-1 & A \\
\hline Ps 776 & ami, gen & azt & pip/taz, pol B & + & SPM-1 & A \\
\hline Ps 780 & cip, ami, gen & azt & pip/taz, pol B & + & SPM-1 & A \\
\hline Ps 783 & cip, ami, gen, pip/taz & azt & pol B & + & SPM-1 & A \\
\hline Ps 790 & cip, ami, gen, pip/taz & azt & pol B & + & SPM-1 & A \\
\hline
\end{tabular}




\begin{tabular}{|c|c|c|c|c|c|c|}
\hline \multirow[b]{2}{*}{$\begin{array}{l}\text { Isolate } \\
\text { number }\end{array}$} & \multicolumn{3}{|c|}{ Resistance profile } & \multirow[b]{2}{*}{$\begin{array}{l}\text { MBL } \\
\text { test }^{a}\end{array}$} & \multirow[b]{2}{*}{$\begin{array}{c}\text { MBL } \\
\text { gene }\end{array}$} & \multirow[b]{2}{*}{$\begin{array}{c}\text { PFGE } \\
\text { pattern }^{b}\end{array}$} \\
\hline & Resistance & $\begin{array}{l}\text { Intermediate } \\
\text { resistance }\end{array}$ & Susceptibility & & & \\
\hline Ps 791 & cip, ami, gen, pip/taz & - & azt, pol B & + & SPM-1 & A \\
\hline Ps 793 & cip, ami, gen, pip/taz & azt & pol B & + & SPM-1 & A \\
\hline Ps 800 & cip, ami, gen & azt & pip/taz, pol B & + & SPM-1 & A \\
\hline Ps 810 & cip, ami, gen, pip/taz & azt & pol B & + & SPM-1 & A \\
\hline Ps 822 & cip, ami, gen, pip/taz & azt & pol B & + & SPM-1 & A \\
\hline Ps 830 & cip, ami, gen, pip/taz & azt & pol B & + & SPM-1 & A \\
\hline Ps 832 & cip, ami, gen & - & pip/taz, azt, pol B & + & SPM-1 & A \\
\hline Ps 842 & cip, ami, gen, pip/taz & - & azt, pol B & + & SPM-1 & A \\
\hline Ps 851 & cip, ami, gen, pip/taz & azt & pol B & + & SPM-1 & A3 \\
\hline Ps 860 & cip, ami, gen, pip/taz & azt & pol B & + & SPM-1 & A \\
\hline Ps 874 & cip, ami, gen & azt & pol B & neg & AT & A4 \\
\hline Ps 883 & cip, ami, gen, pip/taz & azt & pol B & + & SPM-1 & A5 \\
\hline Ps 887 & cip, ami, gen & - & azt, pol B & + & SPM-1 & A6 \\
\hline Ps 900 & cip, ami, gen & - & pip/taz, azt, pol B & + & SPM-1 & $\mathrm{A}$ \\
\hline $2008 / 2009$ & & - & & & & \\
\hline Ps 901 & cip, ami, gen, pip/taz azt & - & pol B & neg & AT & $\mathrm{B}$ \\
\hline Ps 902 & cip, ami, gen, azt & - & $\mathrm{pip} / \mathrm{taz}, \mathrm{pol} \mathrm{B}$ & neg & AT & $\mathrm{C}$ \\
\hline Ps 904 & cip, ami, gen, pip/taz, azt & - & pol B & neg & AT & $\mathrm{D}$ \\
\hline Ps 905 & cip, ami, gen, pip/taz & azt & pol B & + & SPM-1 & A7 \\
\hline Ps 906 & cip, ami, gen, azt & - & $\mathrm{pip} / \mathrm{taz}, \mathrm{pol} \mathrm{B}$ & neg & AT & $\mathrm{D}$ \\
\hline Ps 908 & cip, ami, gen, pip/taz, azt & - & pol B & neg & AT & $\mathrm{E}$ \\
\hline Ps 909 & cip, gen, azt & ami & pol B & neg & AT & $\mathrm{D}$ \\
\hline Ps 912 & cip, ami, gen, pip/taz & azt & pol B & + & SPM-1 & A7 \\
\hline Ps 918 & cip, ami, gen, pip/taz, azt & - & pol B & neg & AT & $\mathrm{F}$ \\
\hline Ps 919 & cip, ami, gen, pip/taz, azt & - & pol B & neg & AT & $\mathrm{G}$ \\
\hline Ps 920 & cip, ami, gen, pip/taz, azt & - & pol B & neg & AT & $\mathrm{F}$ \\
\hline Ps 922 & cip, ami, gen, pip/taz, azt & - & pol B & + & SPM-1 & A \\
\hline
\end{tabular}

$a$ : results showed as positive $(+)$ or negative (neg) for the phenotypic Ambler class B metallo- $\beta$-lactamases (MBL) test; $b$ : according to Tenover et al. (1995); ami: amikacina; AT: absence of tested MBL genes; azt: aztreonam; cip: ciprofloxacin; gen: gentamicin; PFGE: pulsed-field gel electrophoresis; pip/taz: piperacilin/tazobactam; pol B: polymyxin B; SPM: São Paulo metallo- $\beta$-lactamase.

and Information). In addition, all of the isolates were genotyped by DNA macrorestriction followed by pulsedfield gel electrophoresis (PFGE) using the endonuclease SpeI. A representative isolate of the SPM Brazilian clone (from São Paulo Hospital) and the sequenced strain PA01 (kindly provided by the Pseudomonas Genome Project, Boston, MA, USA) were included as a reference point. Clonal relationships among the isolates were established using the criteria of Tenover et al. (1995).

The isolates from the present work revealed an important change in the epidemiology of carbapenem-resistant $P$. aeruginosa isolates between the periods $2002-$ 2003 and 2008-2009, showing a decreasing prevalence of the epidemic SPM-1-producing clone in the final period of bacterial recovery (Table). Moreover, the antimicrobial susceptibility test revealed that these bacteria were co-resistant to many other anti-Pseudomonas drugs, particularly the most recent strains (2008/2009). On the other hand, all of the bacterial samples were susceptible to polymyxin B (Table).
A high prevalence of the MBL phenotype was observed among the 2002/2003 isolates (98.4\%) (Table). This coincided with a higher incidence of $b l a_{\mathrm{SPM}-1}$ than those found in previous studies conducted on a national scale (Sader et al. 2004, Gräf et al. 2008). Nevertheless, this high incidence of the $b l a_{\mathrm{SPM}-1}$ gene decreased in the 2008/2009 isolates (Table), suggesting that carbapenemresistance mechanisms other than MBL must be present and are being spread in the hospital under study. Moreover, these other mechanisms, such as efflux pump over expression (associated or not with porin down-regulation), may also be involved in the overall increased resistance to anti-Pseudomonas drugs observed in 2008/2009. None of the isolates showed amplification of the $b l a_{\mathrm{IMP}}$ or $b l a_{\text {VIM }}$ gene (data not shown). Thus, two presumptive MBL producers, from 2002/2003, did not carry any of the MBL genes tested. As expected, none of the $10 \mathrm{MBL}-$ negative isolates indicated the presence of MBL genes.

Molecular typing indicated the prevalence of bacterial isolates (herein designated as genotype A) closely re- 
lated to the widespread SPM Brazilian clone 48-1997A, which was widely disseminated in the 2002/2003 group of isolates (Table). The existence of common PFGE types among carbapenem-resistant $P$. aeruginosa isolates from distinct geographical locations has been reported and indicates clonal dispersion (Zavascki et al. 2005, Fonseca et al. 2010). In Brazil, there have been previous reports of the spread of a unique SPM-type MBL-positive clone (Gales et al. 2003). As expected, MBL-negative isolates from 2008/2009 were unrelated to the epidemic Brazilian clone and showed six distinct PFGE types (Table). Thus, new clones could be responsible for the dissemination of other resistance mechanisms to carbapenems. The three 2008/2009 MBL-positive isolates that carried the $b l a_{\mathrm{SPM}-1}$ gene belonged to the clonal pattern A. Interestingly, the increase in bacterial variation was also accompanied by an increase in bacterial resistance. It is noteworthy that the MIC values for imipenem and meropenem were not high among the more recent isolates (MIC $>8 \mu \mathrm{g} / \mathrm{mL}$ ), which corroborates the hypothesis that there are alternative resistance mechanisms. This is supported by the fact that MBL enzymes increase antimicrobial MICs more effectively than does either efflux pump over-expression or porin down-regulation alone (Xavier et al. 2010).

In conclusion, the population of carbapenem-resistant $P$. aeruginosa in the hospital under study was replaced by MBL-negative, genetically unrelated bacterial isolates. This finding emphasises the need for continuous surveillance strategies and an improvement of the infection control measures in this institution.

\section{ACKNOWLEDGEMENTS}

To Dr Ana Cristina Gales (Special Clinical Microbiology Laboratory and ALERTA Laboratory, UNIFESP), who kindly provided the isolates used for the SPM, IMP and VIM positive controls in this study.

\section{REFERENCES}

Arakawa Y, Shibata N, Shibayama K 2000. Convenient test for screening metallo-beta-lactamase-producing gram-negative bacteria by using thiol compounds. J Clin Microbiol 38: 40-43.

CLSI - Clinical Laboratory Standards Institute 2010. Performance standards for antimicrobial susceptibility testing 2010, 20th Informational Supplement M100-S20, CLSI, Wayne, $160 \mathrm{pp}$.

Fonseca EL, Freitas FS, Vicente ACP 2010. The colistin-only-sensitive Brazilian Pseudomonas aeruginosa clone SP (sequence type 277) is spread worldwide. Antimicrob Agents Chemoter 54: 2743.
Gales AC, Menezes LC, Silbert S, Sader HS 2003. Dissemination in distinct Brazilian regions of an epidemic carbapanem-resistant Pseudomonas aeruginosa producing SPM metallo- $\beta$-lactamase. $J$ Antimicrob Chemother 52: 699-702.

Gräf T, Fuentefria DB, Corção G 2008. Ocorrência de cepas de Pseudomonas aeruginosa multirresistentes produtoras de metalo- $\beta$-lactamase $b l a_{\mathrm{SPM}-1}$ em amostras clínicas. Rev Soc Bras Med Trop 3: 306-308.

Hurst M, Lamb HM 2000. Meropenem: a review of its use in patients in intensive care. Drugs 3: 653-680.

Migliavacca R, Docquier JD, Mugnaioli C, Amicosante G, Daturi R, Lee K, Rossolini GM, Pagani L 2002. Simple microdilution test for detection of metallo- $\beta$-lactamase production in Pseudomonas aeruginosa. J Clin Microbiol 40: 4388-4390.

Rodríguez-Martínez JM, Poirel L, Nordmann P 2009. Molecular epidemiology and mechanisms of carbapenem resistance in Pseudomonas aeruginosa. Antimicrob Agents Chemoter 53: 4783-4788.

Sader HS, Castanheira M, Mendes RE, Toleman M, Walsh TR, Jones RN 2004. Dissemination and diversity of metallo- $\beta$-lactamases in Latin America: report from the SENTRY Antimicrobial Surveillance Program. Int J Antimicrob Agents 25: 57-61.

Salabi AL, Toleman MA, Weeks J, Bruderer T, Frei R, Walsh TR 2010. First report of the metallo- $\beta$-lactamase, SPM-1, in Europe. Antimicrob Agents Chemother 54: 582.

Strateva T, Yordanov D 2009. Pseudomonas aeruginosa - a phenomenon of bacterial resistance. J Med Microbiol 58: 1133-1148.

Tenover FC, Airbeit RD, Goering RV 1995. Interpreting chromosomal DNA restriction patterns produced by pulsed field gel electrophoresis: criteria for bacterial strain typing. J Clin Microbiol 33: 2233-2239.

Toleman MA, Biedenbach D, Bennett DMC, Jones RN, Walsh TR 2005. Italian metallo- $\beta$-lactamases: a national problem? Report from the SENTRY Antimicrobial Surveillance Programme. J Antimicrob Chemother 55: 61-70.

Xavier DE, Picão RC, Girardello R, Fehlberg LCC, Gales AC 2010. Efflux pumps expression and its association with porin downregulation and $\beta$-lactamase production among Pseudomonas aeruginosa causing bloodstream infections in Brazil. BMC Microbiol 10: 217.

Zavascki AP, Gaspareto PB, Martins AF, Gonçalves AL, Barth AL 2005. Outbreak of carbapenem-resistant Pseudomonas aerugino$s a$ producing SPM-1 metallo- $\beta$-lactamase in a teaching hospital in southern Brazil. J Antimicrob Chemother 56: 1148-1151. 